\title{
Pointwise bounded approximation by polynomials
}

\author{
BY ANTHONY G. O'FARRELL \\ Maynooth College, Co. Kildare, Ireland \\ AND FERNANDO PEREZ-GONZALEZ \\ Department de Análysis Matemático, CLMAC, Universidad de la Laguna, \\ 38271 La Laguna, Tenerife, Canary Islands, Spain
}

(Received 3 June 1991; revised 28 October 1991)

\section{Introduction}

For a bounded open set $U \subset \mathbb{C}$, we denote by $H^{\infty}(U)$ the collection of all bounded analytic functions on $U$. We let $X$ denote bdy $(U)$, the boundary of $U, Y$ denote the polynomial hull of $U$ (the complement of the unbounded component of $\mathbb{C} \backslash X$ ), and $U^{*}$ denote $\operatorname{int}(Y)$, the interior of $Y$. We denote the sup norm of a function $f: A \rightarrow \mathbb{C}$ by $\|f\|_{A}$ :

$$
\|f\|_{A}=\sup \{|f(z)|: z \in A\} .
$$

We denote the space of all analytic polynomials by $\mathbb{C}[z]$, and we denote the open unit disc by $\mathrm{D}$ and the unit circle by $\mathrm{S}^{\mathbf{1}}$.

This paper concerns the possibility of approximating a function $f \in H^{\infty}(U)$, using only polynomials which satisfy the same control as $|f|$ on a prescribed subset $S \subset U$. This kind of approximation was first considered by O. J. Farrell, and has been studied by Rubel and Stray (cf. [4]).

Definition. Let $U \subset \mathbb{C}$ be a bounded open set, and $F \subset \operatorname{clos}(U)$ be closed. We say that $(U, F)$ is a Farrell pair if for each $f \in H^{\infty}(U)$, and each $\epsilon>0$, there exist $p_{m}(z) \in \mathbb{C}[z]$, such that

$$
p_{m}(z) \rightarrow f(z), \quad \text { for all } z \in U,\left\|p_{m}\right\|_{U} \leqslant\|f\|_{U}, \quad \text { and } \quad\left\|p_{m}\right\|_{F} \leqslant\|f\|_{F \cap U}+\epsilon .
$$

We shall characterize the Farrell pairs.

In view of the Farrell-Rubel-Shields Theorem ([2], p. 151), $\mathbb{C}[z]$ is pointwise boundedly dense in $H^{\infty}(U)$ if and only if each $f \in H^{\infty}(U)$ extends to $H^{\infty}\left(U^{*}\right)$, and this in turn happens if and only if there are components $U_{1}, U_{2}, U_{3}, \ldots$, of $U^{*}$ and closed sets $E_{1}, E_{2}, E_{s}, \ldots$, such that

$$
U=\bigcup_{n}\left(U_{n} \backslash E_{n}\right)
$$

and the sets $E_{n} \cap U_{n}$ have inner analytic capacity zero. Thus, if we are studying situations in which $\mathbb{C}[z]$ is pointwise boundedly dense in $H^{\infty}(U)$, then there is no loss in generality in assuming that $U$ is a union of some components of $U^{*}$ (i.e. that the $E_{n}$ are empty).

We call a set $U$ an FRS set if it is open and bounded and is a union of components of its interior $U^{*}$. Note that the components of an FRS set are simply connected.

Since we lose nothing essential, we shall work exclusively with FRS sets $U$.

When $U$ is an FRS set, with components $U_{1}, U_{2}, U_{3}, \ldots$, we fix a point $a_{n} \in U_{n}$ and 
let $\lambda_{n}$ denote harmonic measure for $a_{n}$ on bdy $\left(U_{n}\right)$. We set $\lambda=\sum_{1}^{\infty} \lambda_{n} / 2^{n}$, and call $\lambda$ a harmonic measure for $U$. We note that $\lambda_{n}$ is singular to $\lambda_{m}$ whenever $n \neq m$. (The simplest way to see this is to note, firstly, that $U_{m}$ and $U_{n}$ can share at most one common accessible boundary point, secondly, by Fatou's Theorem, that the harmonic measure for $U_{n}$ is supported on the set of accessible boundary points, and thirdly, that harmonic measure contains no point masses.) There is a canonical isometric injection $f \mapsto \tilde{f}$ of $H^{\infty}(U) \rightarrow L^{\infty}(\lambda)$. There are a number of equivalent ways of describing $\tilde{f}$. One way is to map the $n$th component $U_{n}$ conformally to $\mathrm{D}$, transfer $f \mid U_{n}$ to a function in $H^{\infty}(\mathrm{D})$, take non-tangential limits, a.e. $d \theta$, and transfer them back. This defines $\tilde{f}$ a.e. $d \lambda_{n}$. Since the various $\lambda_{n}$ are mutually-singular, this suffices to define $\tilde{f}$. A second way is to use Brownian motion. For our present purposes, the most relevant is this. We may choose a sequence $p_{m}$ of polynomials converging pointwise boundedly on $U$ to $f$, and converging $\lambda$-a.e. See [2], (VI-5.2). The limit is $\tilde{f}$.

For each $n$, we fix a conformal map $\phi_{n}: U_{n} \rightarrow \mathrm{D}$. Even though $\phi_{n}$ need not extend continuously to $X_{n}=\operatorname{bdy}\left(U_{n}\right)$, it does extend as a Borel bijection of a set of full $\lambda_{n}$ measure on $X_{n}$, onto a set of full $d \theta$ measure on $\mathrm{S}^{1}$. The extension is non-unique, but it is determined up to sets of measure zero at each end. We denote by $H_{n}$ the image in $\mathrm{S}^{1}$ of $F$ under such a bijection.

THEOREM 1. Let $U$ be an FRS set with components $U_{n}(n=1,2,3, \ldots)$ and harmonic measure $\lambda$. Let $F$ be a closed subset of $\operatorname{clos}(U)$. Then the following are equivalent:

(1) $(U, F)$ is a Farrell pair;

(2) $\left(U_{n}, F \cap \operatorname{clos}\left(U_{n}\right)\right)$ is Farrell, for each $n$;

(3) for each $f \in H^{\infty}(U)$,

$$
|\tilde{f}| \leqslant\|f\|_{F \cap U}, \quad \lambda \text {-a.e. on } F
$$

(4) For each $n$, d $\theta$-almost-every point of $H_{n}$ is a non-tangential point of $\phi_{n}\left(F \cap U_{n}\right)$;

(5) for each closed set $A \subset F \cap$ bdy $(U)$, there is a sequence of measures $\mu_{n}(n=1,2$, $3, \ldots)$ with compact support lying in $F \cap U$, and having $\left\|\mu_{n}\right\| \leqslant \lambda(A)$ such that $\mu_{n} \rightarrow \lambda \mid A$, in the weak-star topology of $H^{\infty}(U)^{*}$.

In the case when $U$ is the unit disc, this theorem is due to Stray [4].

Note that the equivalence of (1) and (2) does not state that "there is no interaction between distinct components in this problem '. It takes this neat form because of the formulation in terms of closed sets $F$.

We observe that (5) is equivalent to the same statement, with 'closed set' replaced by 'Borel set'.

We note that it is not true that $(U, F)$ is Farrell if and only if $\left(U^{*}, F\right)$ is Farrell. The latter condition implies the former, but not conversely. The classical cornucopia (Figure 1) provides an example.

An example with smoothly bounded components is provided by the budding disc (Figure 2).

Letting $U$ denote the union of the buds,

$$
U=\bigcup_{p=2}^{\infty} \bigcup_{j=0}^{2^{p}}\left\{x \in \mathbb{C}:\left|z-a_{p, j}\right|<2^{-2^{p}}\right\}
$$

where

$$
a_{p, j}=\left(1+2^{-2^{p}}\right) \exp \left(2 \pi j / 2^{p}\right)
$$




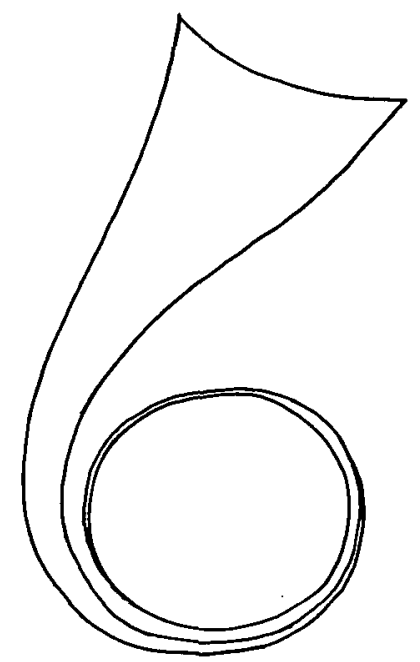

Fig. 1. The cornucopia.

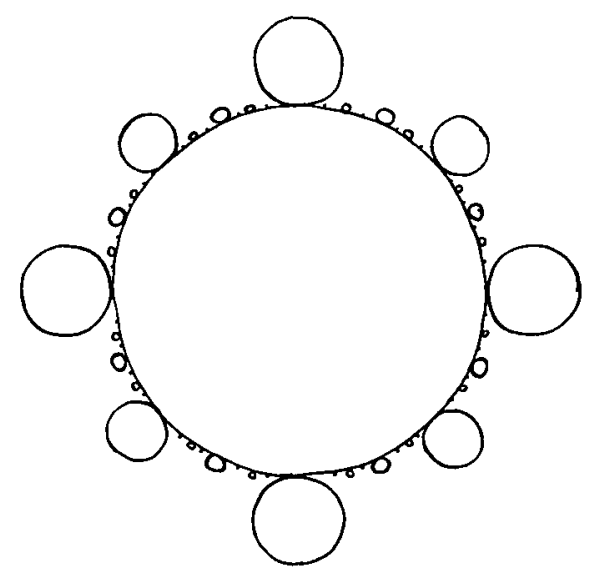

Fig. 2. The budding disc.

we find that $U^{*}$ is obtained from $U$ by adding the open unit disc. Taking

$$
S=\left\{a_{p, j}: p \geqslant 2,0 \leqslant j \leqslant 2^{p}\right\},
$$

and $F=\operatorname{clos}(S)$, we see that $(U, F)$ is once again Farrell, while $\left(U^{*}, F\right)$ is not.

\section{Proof of Theorem 1}

We begin with a lemma about FRS sets.

LEMMA 1. If $V$ is a union of components of an FRS set, then $V$ is itself an FRS set.

Proof. Let $u$ be an FRS set, with components $U_{1}, U_{2}, U_{3}, \ldots$, and let

$$
V=\bigcup_{n \in J} U_{n}
$$

be a union of components of $U$. We must prove that for each $n \in J$ the set $U_{n}$ is a component of $V^{*}$. 
Fix $n \in J$, and let $W$ be the component of $V^{*}$ that contains $U_{n}$. If $W \neq U_{n}$, then there exists a point

$$
a \in W \cap \operatorname{bdy}\left(U_{n}\right) \subset V^{*} \cap \operatorname{bdy}\left(U_{n}\right) .
$$

But then $a \in \operatorname{bdy}\left(U^{*}\right)$, since bdy $\left(U_{n}\right) \subset$ bdy $\left(U^{*}\right)$ and $U$ is FRS. Since $\mathbb{C} \backslash \operatorname{clos}\left(U^{*}\right)$ is a subset of $\mathbb{C} \backslash V$, this implies that $a \in \operatorname{clos}\left(\mathbb{C} \backslash V^{*}\right)$, contradicting the openness of $V^{*}$. Thus $W=U_{n}$.

Now we begin the proof of Theorem 1 . We use the notation

$$
S=F \cap U, \quad X_{n}=\operatorname{bdy}\left(U_{n}\right), \quad F_{n}=F \cap \operatorname{clos}\left(U_{n}\right), \quad S_{n}=F \cap U_{n} .
$$

$(1) \Rightarrow(2)$ Suppose that $(U, F)$ is a Farrell pair. Fix $n$. Let $f \in H^{\infty}\left(U_{n}\right)$ and $\epsilon>0$ be given. We have to show that we may approximate $f$ pointwise on $U_{n}$ by polynomials $p_{m}$ satisfying

$$
\left\|p_{m}\right\|_{U_{n}} \leqslant\|f\|_{U_{n}} \text { and }\left\|p_{m}\right\|_{F_{n}} \leqslant\|f\|_{S_{n}}+\epsilon
$$

Extend $f$ to an element of $H^{\infty}(U)$ by defining $f=0$ on the remaining components $U_{m}(m \neq n)$ of $U$. Then $\|f\|_{U}=\|f\|_{U_{n}}$ and $\|f\|_{F}=\|f\|_{F_{n}}$, so the existence of the required polynomials $p_{m}$ follows from the fact that $(U, F)$ is Farrell.

$(2) \Rightarrow(3)$ This implication may be proved component be component. Further, since, by Lemma 1, the components of an FRS set are themselves FRS sets, we may suppose that $U$ is connected for this part of the proof.

So let $U$ be a connected FRS set and $F \subset \cos (U)$ be such that $(U, F)$ is Farrell. We must show that the extension $\tilde{f}$ in $L^{\infty}(\lambda)$ has $|\tilde{f}| \leqslant\|f\|_{S}, \lambda$-a.e. This is implicitly proved in Stray's paper, in the case when $U=\mathrm{D}$, but we cannot simply reduce to that case, because the conformal map from $U$ to $D$ may fail to extend continuously (FRS examples exist; see Section 3, below), hence there is no simple correspondence of Farrell pairs $(U, F)$ and $\left(\mathrm{D}, F^{\prime}\right)$. Here is a direct argument, based on Stray's idea.

Fix $f \in H^{\infty}(U)$ and $\eta>\|f\|_{S}$. Suppose (for a contradiction) that there exists a compact set $A \subset F$, with $\lambda(A)>0$, on which $|f|>\eta$. By means of obvious modification to $f, \eta$ and $A$, we may arrange that

and

$$
\|f\|_{S} \leqslant \eta<\mathbf{1}=\|f\|_{U}
$$

$$
\text { the real part } \Re f \text { of } f \text { exceeds } \frac{1}{2}(\eta+1) \text { on } A \text {. }
$$

Let $p_{m} \in \mathbb{C}[z]$ be any sequence converging pointwise to $f$ on $U$, with $\left\|p_{m}\right\|_{U} \leqslant 1$. We claim that necessarily $\lim \inf \left\|p_{m}\right\|_{F}>\eta$.

By Fatou's Lemma, we may choose $z \in U$ such that the harmonic measure $v$ with respect to $z$ of $A$ exceeds $\frac{1}{4}(3+\eta)$ and $\mathfrak{R} f(z)>\frac{1}{2}(\eta+1)$. Then, for large enough $m$, we get $\left|p_{m}(z)\right|>\frac{1}{2}(\eta+1)$. But

$$
\left|p_{m}(z)\right| \leqslant \int_{A}\left|p_{m}(\zeta)\right| d v(\zeta)+\int_{X \backslash A}\left|p_{m}(z)\right| d v(\zeta) \leqslant\left\|p_{m}\right\|_{A}+\frac{1}{4}(1-\eta),
$$

and this yields the claim.

$$
\left\|p_{m}\right\|_{A}>\frac{1}{4}(3 \eta+1),
$$

This contradicts the assumption that $(U, F)$ is Farrell. Thus (2) implies (3).

$(3) \Rightarrow(1)$ We resume the consideration of the general FRS set $U$. Suppose that (3) holds. We must show that $(U, F)$ is Farrell.

Let $f \in H^{\infty}(U)$ and $\epsilon>0$ be given. Let $\eta=\|f\|_{S}+\epsilon$. We will modify an argument that goes back to Davie to show that $f$ may be approximated pointwise on $U$ by polynomials $p_{m}$ that have $\left|p_{m}\right| \leqslant 1$ on $U$ and $\left|p_{m}\right| \leqslant \eta$ on $\boldsymbol{F}$. 
First, we extend $f$ to $U^{*}$ by defining $f=0$ on $U^{*} \backslash U$. This makes $f$ an element of $H^{\infty}\left(U^{*}\right)$.

Let $\lambda^{*}$ denote a harmonic measure for $U^{*}$ on $X$. Then $\lambda \ll \lambda^{*}$.

Let

$$
N=\left\{g \in C(Y):\|g\|_{F} \leqslant \eta, \quad\|g\|_{Y} \leqslant 1\right\} .
$$

Here $C(Y)$ denotes the uniform algebra of all continuous complex-valued functions on $Y$.

By Mergelyan's Theorem [2], the closure of $\mathbb{C}[z]$ in $C(Y)$ is the algebra $A(Y)$ of all those $g \in C(Y)$ that are analytic on $U^{*}$. Thus it is enough for us to show that $f$ belongs to the closure, in the topology of uniform convergence on compact subsets of $U$, of the set $N \cap A(Y)$. This is a locally-convex topology, so we may apply the Separation Theorem. Further, the continuous linear functionals on the space of analytic functions on $U$, with the topology of uniform convergence on compact sets, are represented by measures having compact support on $U$. Thus all we need show is that

$$
\left|\int f d \mu\right| \leqslant 1
$$

whenever $\mu$ is such a measure and satisfies

$$
\left|\int h d \mu\right| \leqslant 1
$$

for all $h \in N \cap A(Y)$.

Let $\mu$ have these properties. The set $N$ is the unit ball for an equivalent norm on the space $C(Y)$. Thus we may apply the Hahn-Banach Theorem and the Riesz Representation Theorem to obtain a measure $\nu$, supported on $Y$, such that

$$
\begin{gathered}
\left|\int g d \nu\right| \leqslant 1, \quad \text { for all } g \in N \\
\int h d \nu=\int h d \mu, \quad \text { for all } h \in N \cap A(Y) .
\end{gathered}
$$

It follows that$$
|\nu|(Y \backslash F)+\eta|\nu|(F) \leqslant 1 .
$$

We claim that $\nu \mid X$ is absolutely continuous with respect to $\lambda^{*}$. In fact, let $\mu_{1}$ be the balayage of $\mu$ to $X$, and let $\nu_{1}$ be the balayage of $\nu \mid U$. Then $\mu_{1}$ and $\nu_{1}$ are absolutely continuous with respect to $\lambda^{*}$, and $\mu_{1}-v_{1}-(\nu \mid X)$ annihilates $A(Y)$. By a generalized F. and M. Riesz Theorem ([2], theorem II.8.6), the function $\mu_{1}-\nu_{1}-(\nu \mid X)$ is absolutely continuous with respect to $\lambda^{*}$, hence so is $\nu \mid X$.

Thus $\tilde{f}$ is defined $\nu$-a.e., and we have that $|\tilde{f}| \leqslant \eta$, $\nu$-a.e. on $f$, and $|\tilde{f}| \leqslant 1$, $\nu$-a.e. on $Y$.

Choose $g_{m} \in A(Y)$ such that $g_{m} \rightarrow f$ pointwise $\lambda^{*}$-a.e. on bdy $(Y)$ and $\left\|g_{m}\right\|_{Y} \leqslant 1$. This is possible, by the Farrell-Rubel-Shields Theorem and the Reduction of Norm Theorem ([2], (VI:5-3)). Then

$$
\left|\int f d \mu\right|=\left|\int f d \nu\right| \leqslant\left|\int_{Y \backslash F} f d \nu\right|+\left|\int_{F} f d \nu\right| \leqslant 1 .|\nu|(Y \backslash F)+\eta|\nu|(F) \leqslant 1,
$$

as required. Thus (3) implies (1). 
Before completing the proof, we give a second lemma.

Lemma 2. Let $H \subset \mathrm{S}^{1}$ and $S \in \mathrm{D}$ be Borel sets. Then the following are equivalent:

(1) $d \theta$-almost every point of $H$ is a non-tangential limit point of $S$;

(2) for each Borel set $T \subset H$ there is a sequence of measures $\mu_{n},(n=1,2,3, \ldots)$ with compact support lying in $S$, and having total mass less than or equal to the $d \theta$-measure $|T|$ of $T$, such that $\mu_{\nu} \rightarrow d \theta \mid T$, in the weak-star topology in $H^{\infty}(\mathrm{D})^{*}$.

(3) $|\tilde{f}| \leqslant\|f\|_{S} d \theta$-a.e. on $H$, for each $f \in H^{\infty}(\mathrm{D})$. (Here $\tilde{f}$ denotes the non-tangential limit of $f$.)

In formulating condition (2), we take the point of view that $d \theta \mid E$ acts on $H^{\infty}(\mathrm{D})$ via the usual (Fatou) map $f \mapsto \tilde{f}, H^{\infty}(\mathrm{D}) \rightarrow L^{\infty}(d \theta)$.

Note in particular that condition (2) does not just say that $\mu_{n} \rightarrow d \theta \mid E$ in the usual weak-star topology on measures (i.e. weak-star in $C(\operatorname{clos}(\mathrm{D})))$ ). That would not be sufficient, and (2) is a much stronger statement.

It is not hard to see that it is equivalent to demand condition (2) only for closed sets $T \subset H$.

Proof of Lemma 2. (1) $\Rightarrow$ (2) Assume that almost every point of $H$ is a nontangential limit point of $S$, and let $T$ be a closed subset of $H$. For $\alpha>0$, let us say that $z \in \mathrm{S}^{1}$ is an $\alpha$-non-tangential limit point of $S$ if there is a sequence $z_{n} \in S$, converging to $z$, such that

$$
\alpha .\left|z-z_{n}\right| \leqslant 1-\left|z_{n}\right| \text {. }
$$

We may assume that there exists $\alpha>0$ such that each point of $T$ is an $\alpha$-nontangential limit point of $S$, because the general $T$ may be written as a disjoint union

$$
T=\bigcup_{m=1}^{\infty} T_{m}
$$

where

$$
\bigcup_{m=1}^{p} T_{n}
$$

takes up all but the $2^{-p}$-th part of the measure of $T$, and all points of $T_{m}$ are $\alpha_{m}$-nontangential limit points of $S$ for some $\alpha_{m}>0$. Given suitable sequences $\nu_{m, n}$ for $T_{m}$, their term-by-term sum does the trick for $T$.

For $s \in S$, let $W_{s}$ denote the arc of $S^{1}$

$$
W_{s}=\left\{z \in \mathrm{S}^{1}: \alpha \cdot|s-z| \leqslant 1-|s|\right\} .
$$

For each $n$, take $r=(n-1) / n$. Then $r<1$, and the $\operatorname{arcs} W_{s}$ corresponding to $s \in S$ with $|s|>r$ give a fine covering of $T$, and so we may select points $s_{m} \in S$ $(m=1,2,3, \ldots)$ with $\left|s_{m}\right|>r$ such that the corresponding $W_{s_{m}}$ are pairwise disjoint and cover $d \theta$-almost-all of $T$. Choose an integer $M$ such that

$$
\bigcup_{m=1}^{M} W_{s_{m}}
$$

has all but a proportion $1-r$ of the $d \theta$-measure of $T$. Define $v_{n}$ to be the atomic measure having mass $\left|W_{s_{m}}\right|$ at the point $s_{m}$. Thus $\nu_{n}$ is a finite sum of point masses, supported on $S$.

It follows easily, using the fact that functions belonging to $H^{\infty}(\mathrm{D})$ have nontangential limits a.e. $d \theta$, that $\nu_{n} \rightarrow d \theta|T|$ in the weak-star topology on $H^{\infty}(\mathrm{D})$. 


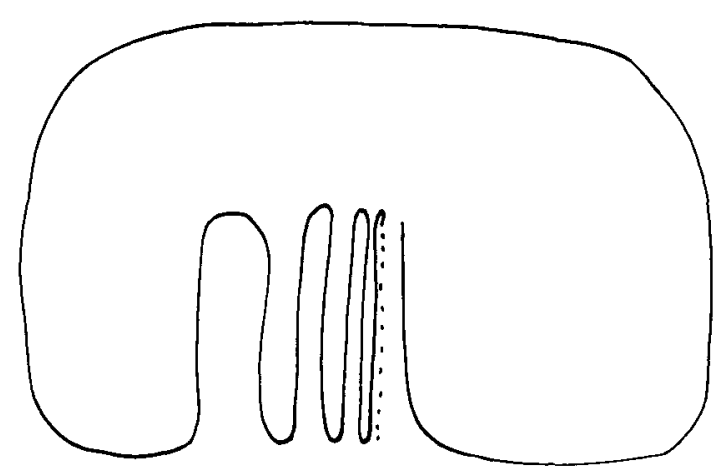

Fig. 3.

$(2) \Rightarrow(3)$ Suppose that (2) holds. If some $f \in H^{\infty}(\mathrm{D})$ satisfied $|\tilde{f}|>\|f\|_{S}$, then we could choose a closed set $T \subset H$, a unimodular constant $\omega$ and a positive $\epsilon$ such that $\mathfrak{R}(\omega f)>\|f\|_{S}+\epsilon$ on $T$. Taking $\nu_{n}$ as in (2), we would then have

$$
\left|\int f d v_{n}-\int_{T} \tilde{f} d \theta\right| \geqslant \epsilon \cdot|T|>0
$$

for all $n$, contradicting the weak-start convergence.

$(3) \Rightarrow(1)$ This was proved by Stray [4], p. 302, in the course of proving his theorem.

We note from the proof that the measures $\nu_{n}$ may be taken discrete, if that is desired.

We now continue the proof of 'Theorem 1.

$(4) \Leftrightarrow(3)$ Since each function in $H^{\infty}\left(U_{n}\right)$ extends to a function in $H^{\infty}(U)$, condition (3) of Theorem 1 is equivalent to the condition that for each $n$ and each $g \in H^{\infty}(\mathrm{D})$, the non-tangential boundary values $\tilde{g}$ on $\mathbf{S}^{1}$ satisfy

$$
|\tilde{g}| \leqslant\|g\|_{\phi_{n}\left(S_{n}\right)}, \quad d \theta \text {-a.e. on } H_{n}
$$

This is because with $f=g \circ \phi_{n}$, we get $\tilde{f}=\tilde{g} \circ \phi_{n}$ a.e. $\lambda_{n}$.

Thus the equivalence of conditions (3) and (4) of the Theorem follows from the equivalence of conditions (1) and (3) of Lemma 2.

$(5) \Leftrightarrow(3)$ In the same way, the equivalence of conditions (1) and (2) of Lemma 2 shows that condition (3) of Theorem 1 is equivalent to the following statement:

for each $n$ and for each Borel set $T \subset H_{n}$ there is a sequence of measures $\mu_{m}$, $(m=1,2,3, \ldots)$ with compact support lying in $\phi_{n}\left(S_{n}\right)$, and having total mass less than or equal to the $d \theta$-measure $|T|$ of $T$, such that $\mu_{m} \rightarrow d \theta \mid T$ in the weak-star topology on $H^{\infty}(\mathrm{D})^{*}$.

Since all the terms in this statement are conformally invariant, we obtain the equivalence of condition (5) of the Theorem.

This concludes the proof of the Theorem.

\section{Remarks and further results}

(3.1) Figure 3 shows an example of an FRS set $U$ for which the conformal map from $U$ to $\mathrm{D}$ is not uniformly continuous. 
(3.2) Let $U$ be a FRS set and $F \subset \operatorname{clos}(U)$ be closed.

From the proof of Theorem 1, we see that $(U, F)$ is Farrell if and only if $\left(U^{*}, F^{*}\right)$ is Farrell, where $F^{*}$ is obtained from $F$ by adding to it a set $S_{n}$ in each component $U_{n}$ of $U^{*} \backslash U$ for which the harmonic measure with respect to $U_{n}$ of $F$ is positive, in such a way that

$$
\operatorname{clos}\left(S_{n}\right)=S_{n} \cup\left(\operatorname{bdy}\left(U_{n} \cap F\right)\right) \text { and }\left(U_{n}, \operatorname{clos}\left(S_{n}\right)\right) \text { is a Farrell set. }
$$

(3.3) Let $U=\bigcup U_{n}$ be an FRS set and $S \subset U$. If $(U$, clos $(S))$ is Farrell, then there does not exist $n$ such that

$$
\lambda_{n}\left(\cos (S) \backslash \operatorname{clos}\left(U_{n} \cap S\right)\right)>0 .
$$

For if there were such an $n$, we could choose a function $u$, harmonic on $U_{n}$, with

$$
\begin{aligned}
& u=0 \text { near } \operatorname{clos}\left(U_{n} \cap S\right) \cap \text { bdy }\left(U_{n}\right), \\
& u=1 \text { on a set of positive } \lambda_{n} \text { measure on } \operatorname{clos}(S), \text { and } \\
& 0<u<1 \text { on } U_{n} .
\end{aligned}
$$

Defining $f=\exp \left(u+\iota u^{*}\right)$ on $U_{n}$, and $f=0$ on the remaining components of $U$, we would have

but

$$
\|f\|_{U}=1, \quad\|f\|_{S}=\|f\|_{S \cap U_{n}}<1,
$$

Thus condition (3) of Theorem 1 would fail, with $F=\operatorname{clos}(S)$, hence $(U, \operatorname{clos} S)$ is not Farrell.

(3.4) In practical terms, if it is desired to check that some pair is a Farrell pair, then (4) and (5) of Theorem 1 are the most readily verified.

It is not, in general, possible to phrase the conditions in terms of non-tangential approach, in the original set $U$. Non-tangential approach (approach from a sector) is not a conformally invariant idea, and (as is known) there may be almost no points on bdy $\left(U_{n}\right)$ that are non-tangentially approachable.

Condition (5) has the advantage that it does not refer to the conformal maps, about which little may be known. It has the disadvantage that weak-star convergence with respect to $H^{\infty}(U)$ may be difficult to check. In general, it is fair to say that the sets for which the conformal maps are ill-behaved are the same as those for which the weak-star convergence is hard to check, so there is little to choose between the two conditions.

$(3 \cdot 5)$ The property of being a Farrell pair is not topological, i.e. there exist pairs $(U, F)$ and $(V, G)$, mapped one to the other by a global homeomorphism of $\mathbb{C}$, such that $(U, F)$ is Farrell and $(V, G)$ is not. To make such an example, modify the budding disc in such a way that the closure of the set of buds meets the unit circle (the boundary of the inner disc) only on a subset $K$ that is perfect and has length zero. Take $U$ to be the union of the central disc and the set of all the buds, and $S$ to be the set of buds. Let $F=S \cup K$. Then $(U, F)$ is a Farrell pair, since $K$ has harmonic measure zero for all components of $U$. We may construct a homeomorphism of $\mathbb{C}$ onto itself, of the form

$$
\Psi: r \exp (\iota \theta) \mapsto r \exp (\iota \psi(\theta)),
$$

where $\psi$ is a periodic homeomorphism of $\mathbb{R}$ onto itself, with the property that $\Psi(K)$ has positive length. The result is that $(\Psi(U), \Psi(K))$ is not Farrell, because condition (3) of Theorem 1 fails. In fact, one can see that the characteristic function of the set of buds cannot be suitably approximated by polynomials. 
(3.6) If the boundary of $U$ is locally smooth off an exceptional set which for each $n$ is the union of a set of Hausdorff dimension less than 1 and a countable union of impressions of prime ends of $U_{n}$, then (by Makarov's Theorem [3]) each $\lambda_{n}$ is mutually absolutely continuous with respect to length (1-dimensional Hausdorff measure) and (by a well known result on conformal mapping) non-tangentiality transfers faithfully under the conformal map $\phi_{n}$, so that we can then rephrase the property of being a Farrell pair in terms of non-tangential limits. This covers all but extremely pathological examples. However, it must be said that extremely pathological examples are generic in plane topology. For instance, one can find three simply connected open sets with the same boundary.

(3.7) Another consequence of Makarov's Theorem is that if $F$ is a compact set of Hausdorff dimension less than 1 , then $(U, K)$ is a Farrell pair whenever $U$ is a FRS set whose closure contains $K$.

(3.8) The property of being a Farrell pair can be rephrased in terms of the maximal ideal space $M$ (or character space) of the uniform algebra $H^{\infty}(U)$. Here is a brief summary of the relevant facts. There is a continuous projection $\pi: M \rightarrow \mathbb{C}$ defined by $\pi(\phi)=\phi(z \mapsto z)$. The algebra $H^{\infty}(U)$ is isometrically isomorphic to the $l^{\infty}$ orthogonal direct sum of the uniform algebras $H^{\infty}\left(U_{n}\right)$. For each $n$, we may consider the maximal ideal space $M_{n}$ of $H^{\infty}\left(U_{n}\right)$. There is usually more to $M$ than the disjoint union of the $M_{n}$, but the rest of it will not concern us.

The harmonic measure $\lambda_{n}$ (for the fixed point $a_{n} \in U_{n}$ ) on $X_{n}$ lifts to $M_{n}$ in many ways, but in one special way. The Shilov boundary $\mathrm{Sh}_{n}$ of $M_{n}$ may be identified with the Choquet boundary of the space of bounded harmonic functions on $U_{n}$; there is a unique probability measure $\lambda_{n}^{*}$ on $\mathrm{Sh}_{n}$ such that

$$
\int_{\mathrm{Sh}_{n}} h(x) d \lambda_{n}^{*}(x)=h\left(a_{n}\right)
$$

whenever $h$ is a bounded harmonic function on $U_{n}$; evidently $\pi_{*} \lambda_{n}^{*}=\lambda_{n}$.

THEOREM 2. Under the hypotheses of Theorem 1, the following are equivalent:

(1) the pair $(U, F)$ is a Farrell pair;

(2) for each $f \in H^{\infty}(U)$ and each $n$, we have $|f(\phi)| \leqslant\|f\|_{F \cap U}$ for $\lambda_{n}^{*}$-almost-all $\phi \in \pi^{-1}(F)$

(3) for each $n, \lambda_{n}^{*}$-almost-all points of $\pi^{-1}(F)$ belong to the weak-star closure in $H^{\infty}(U)^{*}$ of $U \cap F$.

Apart from the considerably enhanced level of abstraction, the proof of this is not essentially different from that of the theorems about $\lambda_{n} \mathrm{~s}$.

This research was partially supported by EOLAS grant SC/90/070 and DGYICT Proyecto PS89-0135.

\section{REFERENCES}

[1] S. D. Fish ER. Function Theory on Planar Domains (John Wiley \& Sons, 1983).

[2] T. W. Gamelin. Uniform Algebras (Prentice-Hall, 1969).

[3] N. G. Makarov. On the distortion of the boundary sets under conformal mapping. Proc. London Math. Soc. (3) 51 (1985), 369-384.

[4] A. Stray. Pointwise bounded approximation by functions satisfying a side condition. Pacific J. Math. 51 (1974), 301-305. 